\title{
Poster: Maintaining UAV Stability using Low-Power WANs
}

\author{
Akshay Gadre \\ Carnegie Mellon University \\ Pittsburgh, Pennsylvania \\ agadre@andrew.cmu.edu
}

\author{
Revathy Narayanan \\ Indian Institute of Technology \\ Madras, Chennai, India \\ revathyn@cse.iitm.ac.in
}

\author{
Swarun Kumar \\ Carnegie Mellon University \\ Pittsburgh, Pennsylvania \\ swarun@cmu.edu
}

\begin{abstract}
Future urban spaces are expected to see Unmanned Aerial Vehicles (UAVs) deployed for wide-ranging applications including product delivery, imaging and search-and-rescue. Yet today's UAVs rely critically on inertial sensors and GPS to remain stable in-flight, meaning they struggle to penetrate urban canyons where GPS is unavailable.

This poster presents LoRaTilt, a system that maintains stability of a UAV using a Low-Power Wide-Area Network (LP-WAN) transmitter. We mount a light-weight and tenyear battery-powered LP-WAN transmitter on a UAV and process its signals from base stations up to hundreds of meter away. We demonstrate how our system estimates and corrects for UAV drift in GPS-denied settings with minimal impact on the cost and flight life of a UAV. Our proof-ofconcept experiments show a promising median accuracy of $1.2326 \mathrm{~mm}$ and 0.0164 radians in estimating the displacement and orientation of a drone with a LoRa LP-WAN radio.
\end{abstract}

\section{KEYWORDS}

\section{LP-WAN; UAV stability; DTW; Phase Synchronization}

\section{ACM Reference Format:}

Akshay Gadre, Revathy Narayanan, and Swarun Kumar. 2018. Poster: Maintaining UAV Stability using Low-Power WANs. In The 24th Annual International Conference on Mobile Computing and Networking (MobiCom '18), October 29-November 2, 2018, New Delhi, India. ACM, New York, NY, USA, 3 pages. https://doi.org/10.1145/3241539. 3267747

\section{INTRODUCTION}

Unmanned Aerial Vehicles (UAVs) are leading candidates in urban spaces for wide-ranging applications from product

Permission to make digital or hard copies of part or all of this work for personal or classroom use is granted without fee provided that copies are not made or distributed for profit or commercial advantage and that copies bear this notice and the full citation on the first page. Copyrights for thirdparty components of this work must be honored. For all other uses, contact the owner/author(s).

MobiCom '18, October 29-November 2, 2018, New Delhi, India

() 2018 Copyright held by the owner/author(s).

ACM ISBN 978-1-4503-5903-0/18/10.

https://doi.org/10.1145/3241539.3267747

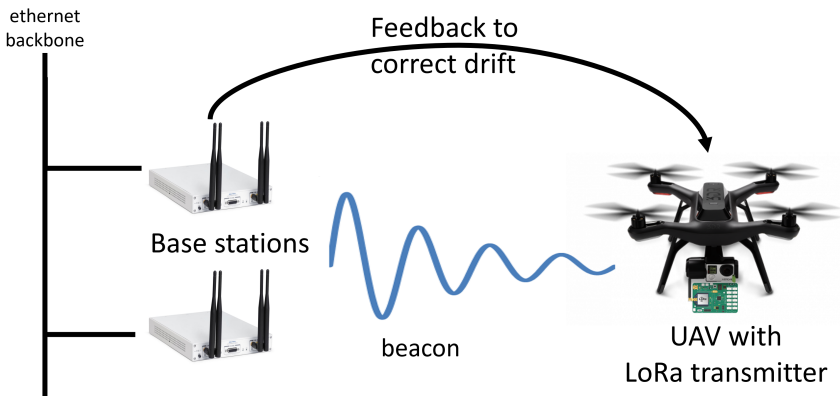

Figure 1: System Diagram of LoRaTilt

delivery (e.g. Amazon Prime Air) to imaging and search-andrescue. Among the most important challenges that UAVs face in urban spaces is their ability to ensure stability as they move or simply hover at a given location. Traditional UAVs do this in rural and suburban spaces using GPS and their on-board inertial sensors to automatically correct for drift in location and orientation. However, UAVs must operate reliably in GPS-denied environments such as tunnels, bridges and urban canyons. Relying on on-board sensors alone has been shown to create gradual drifts over time [6]. This leads to a fundamental challenge of providing accurate stability for UAVs without using heavy or expensive sensors that drain a lot of battery, reducing the flight time.

This poster presents LoRaTilt, a solution that uses wireless signals from a low-power, low-cost transmitter aboard the UAV to estimate it's stability. Specifically, we use LoRa, a LP-WAN technology, that provides a transmission range of kilometers, a lifetime of ten years on a AA battery and will be available as stick-on tags. LoRaTilt processes phase measurements of the wireless signal from the transmitter to base stations as the UAV moves and tilts to estimate the stability of the UAV.

The rest of this paper describes how we overcome challenges in implementing LoRaTilt. First, we must compute the displacement of a LoRa transmitter in rich multipath. LoRaTilt develops a novel algorithm that dynamically learns the phase variation from multipath as the UAV drifts from one location to another. We then use a dynamic time warping(DTW) model that controls the UAV to experience the exact reverse pattern of phase variation and backtrack to return to its initial position. We show how our algorithm remains 
immune to hardware imperfections of LP-WAN transmitters such as frequency offset and timing offset. We use a second transmitter to measure and correct for the orientation of the UAV in the horizontal plane.

We implement a proof-of-concept system on a Holy Stone HS100 UAV, SX1276 SemTech LoRaWAN transmitters and USRP N210 LP-WAN base stations that are about $100 \mathrm{~m}$ away. Our results demonstrate a median $1.2326 \mathrm{~mm}$ accuracy in displacement and 0.0164 radians in orientation of the UAV.

\section{RELATED WORK}

Related work can be broadly categorized as follows:

UAV stability monitoring: There has been much work done on estimating UAV stability using inertial sensors and developing extensive mathematical models[4]. Some have used vision-based sensors[2], which however are limited to line-of-sight. The closest work to ours is [7] which uses the effect of UAV instability on WiFi signals to identify the drone.

UAV and wireless sensing: Past work has developed adhoc sensing solutions for UAV communication [5], collision avoidance and group formations [3]. Other solutions use delays of the wireless network to provide a model that relates the UAV stability with the signal propagation delay[8].

Low-Power WANs: LP-WANs, with their long range and high battery life have been proposed for large-scale applications in rural and urban deployments for agriculture [9] and wildlife monitoring [1], to name a few.

In summary, past work on UAV stability monitoring remains constrained to line-of-sight, operates in limited range, remains vulnerable to drift or reduces flight time. In contrast, we present a solution that operates over long-range, is lightweight and low-power, having minimal effect on the flight time of the UAV.

\section{LORATILT}

LoRaTilt ensures the stability of UAV from large distances by monitoring phase changes in wireless signals received across multiple synchronized base stations. Our key challenge is to accurately model the drift of the UAV in the presence of multipath. Complex multipath causes any displacement or change in orientation of the UAV to result in non-linear phase shifts. At any location, both the multipath characteristics and the UAV's direction of drift are unknown, making estimating the latter very challenging. To make matters worse, multipath characteristics change across locations, meaning that one cannot simply fingerprint the environment to account for it. Modeling Drift from Phase Patterns: LoRaTilt overcomes this challenge by observing that phase variation a UAV experiences as it drifts from one location to the next would occur in reverse should it head backwards along the exact same trajectory. In other words, the UAV at each point in time should measure the wireless channel $\left(\tilde{h}_{1}\right)$ and observe any change in phase $(\vec{h})$. If a phase change occurs over time, the UAV should head along directions that recreate a reverse pattern of the previously observed phase $\operatorname{shifts}\left(\vec{h}_{\text {rev }}\right)$.

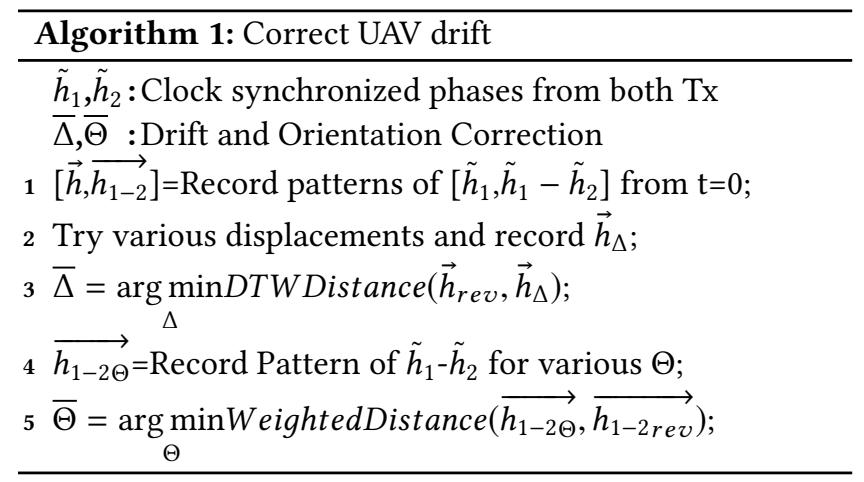

Surprisingly, this approach works well in both line-ofsight and non-line-of-sight environments and is particularly suited to multipath-rich settings. Wireless channels are periodic in line-of-sight, and rotate from 0 to $\pi$ and onto $2 \pi$ in a distance of about a wavelength. This means that any drift lower than half a wavelength (about $15 \mathrm{~cm}$ for LoRa) can be corrected by LoRaTilt. In contrast, wireless channels in multipath-rich settings vary arbitrarily and are likely to experience unique patterns from one wavelength to the other. This means that LoRaTilt in multipath-rich settings can account for greater drift than LoS settings, due to seperable phase change patterns.

A key challenge remains: how do we account for differences in speed of the UAV as it moves forward vs. backward? This means that the phase pattern the base stations perceive from the UAV in reverse maybe stretched or squeezed relative to that observed when the UAV drifted forwards. Our solution relies on dynamic time warping (DTW), a patternrecognition metric that is immune to temporal squeezing and stretching. We measure the DTW-metric for phase patterns observed for four orthogonal directions of the UAV movements relative to the pattern observed as the UAV moved forward. We then move the drone along the direction $(\bar{\Delta})$ that minimizes this metric. Since DTW models remain accurate for time-warped displacement, we observe that the UAV returns to its initial location, albeit not with the same speed. Eliminating time-varying phase offsets: Low cost devices are subject to hardware imperfections, which makes them vulnerable to timing and frequency related offsets, often materializing as phase drifts. This leads to time-varying phase errors in wireless channels at a base station from a UAV leading to inaccurate estimation of UAV stability. LoRaTilt eliminates these time-varying offsets by instead calculating 


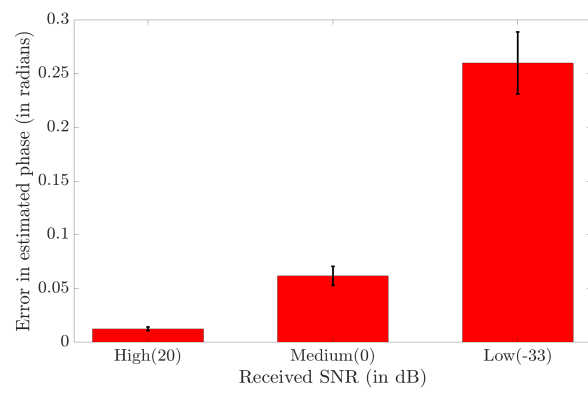

Figure 2: Stability of relative phase

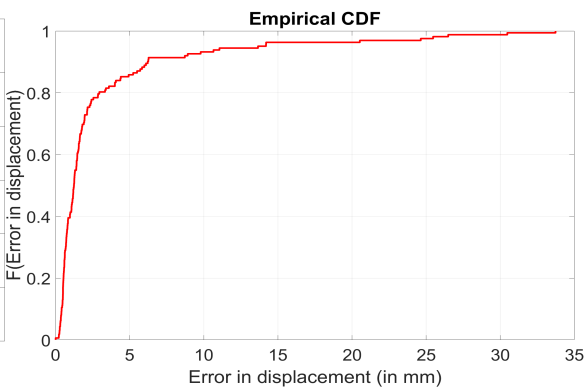

Figure 3: Accuracy of location

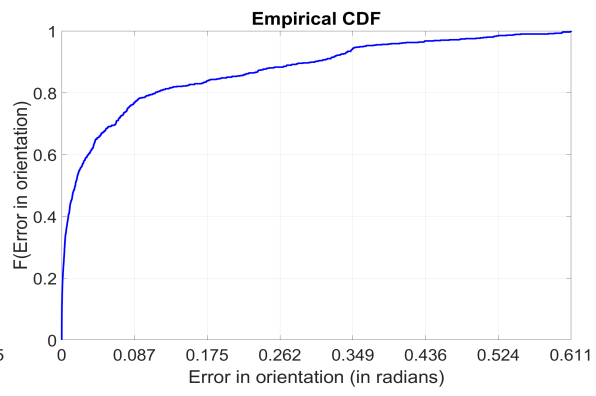

Figure 4: Accuracy of orientation relative phase at two clock-synchronized base stations rather than absolute phase at one base station. Given that LP-WAN base stations are outdoors, we can use GPS-synchronized oscillators to get coordinated phase measurements across radios. Further, although our measurements are still subject to a phase offset due to differences in PLL-locking of the two radios, this offset remains constant as a UAV drift and therefore does not affect our measurements. We note that while relative phase is a different quantity than the phase at one base station, it nevertheless remains stable in the absence of drift and varies in specific patterns along different trajectories in a manner that LoRaTilt can exploit.

Modeling orientation: In addition to displacement, LoRaTilt also measures the orientation of the UAV in the horizontal plane. It measures the relative displacement of a second LoRaWAN transmitter along with the first $\left(\vec{h}_{1-2}\right)$ to estimate the orientation $(\bar{\Theta})$.

\section{PROOF-OF-CONCEPT RESULTS}

We implement LoRaTilt using a SemTech SX1276 transceiver as the LP-WAN transmitter placed on a Holy Stone HS100 UAV and USRP N210s as base stations. We evaluate our system on a test-bed of $10000 \mathrm{~m}^{2}$ that spans urban spaces.

Stability of phase (Fig. 2): We keep the LP-WAN transmitter at various distances from the base stations and plot the variation in phase at a stable location.

Fig. 2 shows the stability of phase of LP-WAN transmitter at extremely low SNRs is within acceptable range for deployment of the system in active UAV drones.

Accuracy of displacement (Fig. 3): We move the drone to various known displacements in 3-D, surveyed using a Bosch GLM-35 measurement device in a fixed orientation and model the phase variation. We then estimate the displacement based on the phase variation.

Fig. 3 shows that LoRaTilt achieves a median accuracy of $1.2326 \mathrm{~mm}$ in predicting displacement. Note that the error does increase to $\sim 1-3 \mathrm{~cm}$ at distance of $100 \mathrm{~m}$, yet remains small enough for real world use. The key reasons behind the high accuracy at large distances are the robustness of LP-WANs in low SNR scenarios and ability of DTWs to accurately model time-warped data.
Accuracy of orientation (Fig. 4): We capture the accuracy in measuring the drone's orientation across different trajectories. We then estimate the orientation using LoRaTilt based on observed phase variation.

Fig. 4 shows that LoRaTilt achieves a median error in orientation of 0.0164 radians. Key reasons for the high accuracy are removal of constant phase offsets across multiple transmitters and robust phase measurements.

\section{CONCLUSION}

We present LoRaTilt, a system for measuring and ensuring stability of UAVs in urban environments where conventional GPS and sensor-based stability mechanisms fail. It uses LP-WAN which imposes minimal power and weight overhead on the drone's operation. LoRaTilt models and reverse-engineers the variation in phase of the wireless channel between the transmitter and base stations to estimate the orientation and displacement of the UAV and demonstrates a promising median accuracy of $1.2326 \mathrm{~mm}$ and 0.0164 radians.

\section{ACKNOWLEDGMENTS}

The work is supported by the NSF grants 1657318 and 1718435 .

\section{REFERENCES}

[1] Andrade, T., ET AL. A reverse GPS architecture for tracking and location of small objects. In ICL-GNSS (2011).

[2] Chiu, C. C., Eт AL. Vision-Only Automatic Flight Control for Small UAVs. IEEE Transactions on Vehicular Technology (2011).

[3] Gupta, L., ET AL. Survey of Important Issues in UAV Communication Networks. IEEE Communications Surveys Tutorials (2016).

[4] Hemakumara, P., ET AL. Learning UAV stability and control derivatives using Gaussian processes. IEEE Transactions on Robotics (2013).

[5] LIN, X., ET AL. The sky is not the limit: LTE for unmanned aerial vehicles. IEEE Comms. (2018).

[6] LiU, Z., ET AL. Control techniques of tilt rotor unmanned aerial vehicle systems: A review. Chinese fournal of Aeronautics (2017).

[7] Nguyen, P., ET AL. Matthan: Drone presence detection by identifying physical signatures in the drone's RF communication. In MobiSys (2017).

[8] Ossa-Gómez, C., ET AL. Design, construction and fly-by-wireless control of an autonomous Quadrotor helicopter. In Fly by Wireless Workshop, Caneus (2011), IEEE.

[9] VAsisht, D., Et Al. An IoT Platform for Data-Driven Agriculture. In NSDI (2017). 